\title{
Tissue- and Development-specific Expression of Multiple Alternatively Spliced Transcripts of Rat Neuronal Nitric Oxide Synthase
}

\author{
Min Ae Lee, ${ }^{\star}$ Li Cai, ${ }^{\ddagger}$ Norbert Hübner, ${ }^{\S}$ Young Ae Lee, ${ }^{\S}$ and Klaus Lindpaintner ${ }^{\star \ddagger \S}$ \\ *Cardiovascular Division, Department of Medicine, Brigham and Women's Hospital, Harvard Medical School, Boston, Massachusetts \\ 02115; ${ }^{\ddagger}$ Department of Cardiology, Children’s Hospital, Harvard Medical School, Boston, Massachusetts 02115; and ${ }^{\S}$ Max Delbrück \\ Centre for Molecular Medicine, 13122 Berlin, Germany
}

\begin{abstract}
Nitric oxide (NO) functions as an intercellular messenger and mediates numerous biological functions. Among the three isoforms of NO synthase that produce NO, the ubiquitously expressed neuronal NO synthase (nNOS) is responsible for a large part of NO production, yet its regulation is poorly understood. Recent reports of two alternative spliceforms of nNOS in the mouse and in man have raised the possibility of spatial and temporal modulation of expression. This study demonstrates the existence of at least three transcripts of the rat nNOS gene designated $\mathrm{nNOSa}, \mathrm{nNOSb}$, and $\mathrm{nNOSc}$, respectively, with distinct $5^{\prime}$ untranslated first exons that arise from alternative splicing to a common second exon. Expression of the alternative transcripts occurs with a high degree of tissue and developmental specificity, as demonstrated by RNase protection assays on multiple tissues from both fetal and adult rats. Furthermore, terminal differentiation of rat pheochromocytoma-derived PC12 cells into neurons is associated with induction of $\mathrm{nNOSa}$, suggesting, likewise, development- and tissue-specific transcriptional control of nNOS isoform expression. Physical mapping using a rat yeast artificial chromosome clone shows that the alternatively spliced first exons $1 \mathrm{a}, 1 \mathrm{~b}$, and 1c are separated by at least $15-60 \mathrm{~kb}$ from the downstream coding sequence, with exons $1 \mathrm{~b}$ and $1 \mathrm{c}$ being positioned within $200 \mathrm{bp}$ of each other.

These findings provide evidence that the biological activity of nNOS is tightly and specifically regulated by a complex pattern of alternative splicing, indicating that the notion of constitutive expression of this isoform needs to be revised. (J. Clin. Invest. 1997. 100:1507-1512.) Key words: nitric oxide $\cdot$ nitric oxide synthase - alternative splicing • gene expression • transcription
\end{abstract}

\section{Introduction}

Nitric oxide $(\mathrm{NO})^{1}$ is synthesized by nitric oxide synthase (NOS) during the NADPH-dependent conversion of L-argi-

Address correspondence to Klaus Lindpaintner, M.D., Cardiovascular Division, Department of Medicine, Brigham and Women's Hospital, 75 Francis Street, Boston, MA 02115. Phone: 617-732-8174; FAX: 617-264-6830; E-mail: kl@calvin.bwh.harvard.edu

Received for publication 20 March 1997 and accepted in revised form 20 June 1997.

J. Clin. Invest.

(C) The American Society for Clinical Investigation, Inc. 0021-9738/97/09/1507/06 \$2.00

Volume 100, Number 6, September 1997, 1507-1512

http://www.jci.org nine to L-citrulline. Three isoforms of NOS encoded by distinct genes have been identified. The neuronal and endothelial NO synthases (nNOS, eNOS) are calcium/calmodulin dependent; their expression has in the past been viewed as constitutive (1-4). In contrast, the inducible isoform (iNOS) isolated from macrophages is calcium/calmodulin-independent, and expression is induced by cytokine stimulation $(5,6)$. NO is a multifunctional intercellular messenger affecting diverse aspects of mammalian physiology including regulation of vascular tone, macrophage-mediated cytotoxicity, neuronal differentiation, and synaptic plasticity (7). While the transinteraction of cytokine-responsive elements within the promoter region is thought to affect the transcriptional regulation of the iNOS gene (8), the mechanisms by which the ubiquitous expression of the nNOS gene is regulated are unknown.

The highest expression of nNOS in the rat has been demonstrated in the brain, in particular in the cerebellum. The gene is also expressed in skeletal muscle, in the myenteric plexus in the gut, in the macula densa of the kidney, and in the heart $(9,10)$. Based on its widespread expression, nNOS is generally considered to be responsible for the largest proportion of tissue NO synthase activity. Although traditionally labeled constitutive, the expression of nNOS may in fact be tightly regulated, as suggested by a number of recent observations. nNOS expression was found to be subject to regulation by estrogen (11), and was induced after axotomy, in parallel with an increased expression of the transcription factors jun and krox $(12,13)$. Furthermore, transient changes in levels of expression of nNOS have been observed in the developing rat nervous system and lung $(14,15)$. Likewise, the onset of nNOS expression in the developing tectum in chicken coincides with the onset of innervation by axons from retinal ganglion cells (16).

Recently, two alternatively spliced nNOS transcripts arising from two alternative promoters and differing in their $5^{\prime}$-untranslated regions ( $5^{\prime} \mathrm{UTR}$ ) have been described in human and murine nNOS genes $(17,18)$. The use of multiple alternative promoters in conjunction with alternative splicing may represent a mechanism by which spatial as well as temporal regulation of nNOS gene transcription is achieved.

Here we present evidence for the presence of three differentially spliced nNOS variants in the rat as well as a comprehensive analysis of spatial and temporal expression patterns that further supports the notion that nNOS expression is modulated by specific and complex regulatory mechanisms on the transcriptional level.

1. Abbreviations used in this paper: iNOS, inducible nitric oxide synthase; NGF, nerve growth factor; nNOS, neuronal nitric oxide synthase; NO, nitric oxide; NOS, nitric oxide synthase; RACE, rapid amplification of cDNA ends; RT, reverse transcription; UTR, untranslated regions; YAC, yeast artificial chromosome. 


\section{Methods}

Rapid amplification of $c D N A$ ends (RACE). Total RNA was extracted from frozen rat tissues by the guanidium thiocyanate-cesium chloride gradient method. Genomic DNA was removed by incubation with RNase-free DNase (Promega Corp., Madison,WI) followed by phenol/chloroform/isoamyl alcohol (25:24:1) extraction and ethanol precipitation. RACE was carried out as previously described (19). In brief, first-strand cDNA was synthesized from $3 \mu \mathrm{g}$ total RNA of various tissues with primer N1 (5'-ggcgtcatctgctcattccgattc-3') located at position 211 of the published sequence for the rat nNOS gene (3), using Superscript II RNase H- Reverse Transcriptase (GIBCO BRL Life Technologies, Inc., Gaithersburg, MD). The cDNA was columnpurified, tailed with terminal transferase (Life Technologies, Inc.) in the presence of dCTP (Pharmacia Diagnostics AB, Uppsala, Sweden), and then amplified in two sequential rounds of nested PCR in a $25 \mu \mathrm{l}$ volume, containing $200 \mathrm{nM}$ of each deoxynucleotide triphosphate, $2 \mathrm{mM}$ magnesium chloride, $0.1 \mathrm{U}$ Taq polymerase (Promega Corp.), $75 \mathrm{nM}$ of a poly $(\mathrm{G})$-anchor primer, and $200 \mathrm{nM}$ of the gene-specific primers N2 (5'-tgtcccegccccttccttactct-3') or N3 $\left(5^{\prime}\right.$ tcaggggcagcaacgggatgtgtc- $\left.3^{\prime}\right)$, respectively. In each reaction, an initial denaturation for $2 \mathrm{~min}$ at $94^{\circ} \mathrm{C}$ was followed by 35 cycles of $94^{\circ} \mathrm{C}$ for $30 \mathrm{~s}, 58^{\circ} \mathrm{C}$ for $1 \mathrm{~min}$, and $72^{\circ} \mathrm{C}$ for $2 \mathrm{~min}$ and a final extension at $72^{\circ} \mathrm{C}$ for $10 \mathrm{~min}$. Amplicons were fractionated on a $2 \%$ agarose gel, purified, and cloned into the PCRII vector using the TA Cloning Kit (Invitrogen Corp., San Diego, CA). Clones were sequenced with the Taq DyeDeoxy Terminator Cycle Sequencing Kit using primer N3 on an ABI373A automatic sequencer (Applied Biosystems Inc., Foster City, CA). All newly determined sequences have been deposited with the GenBank data base (accession numbers: AF008911, AF008912, AF008913).

RNase protection assay. cDNA clones obtained by RACE-PCR were linearized at appropriate restriction sites at the $5^{\prime}$ end of the insert. Complementary RNAs were transcribed from $1 \mu \mathrm{g}$ plasmid in the presence of $\left[\alpha{ }^{32} \mathrm{P}\right] \mathrm{UTP}$ (DuPont-NEN, Boston, MA) with SP6 RNA polymerase using the MAXIscript kit (Ambion Inc., Austin, TX). Labeled probes were purified over Chromaspin-100 columns (Clontech, Palo Alto, CA). RNase protection assay was carried out using the RPAII kit (Ambion Inc.). In brief, 100,000 cpm of labeled probe was hybridized against $100 \mu \mathrm{g}$ total RNA or tRNA overnight at $45^{\circ} \mathrm{C}$. Samples were digested with RNase A and T1, precipitated, and run on a $5.5 \%$ denaturing polyacrylamide gel.

Cell culture and reverse transcription PCR (RT-PCR). Differentiation of rat pheochromocytoma-derived PC12 cells into neurons was carried out as previously described (20). Cells were plated on polyL-lysine coated dishes in DME supplemented with $10 \%$ horse serum and $5 \%$ FCS (Life Technologies, Inc.) at a density of $5 \times 10^{5}$ cells per $10-\mathrm{cm}$ dish. After $16 \mathrm{~h}$ the medium was changed, and $50 \mathrm{ng} / \mathrm{ml}$ nerve growth factor (NGF; Sigma Chemical Co., St. Louis, MO) was added. Neurite outgrowth development was monitored by light microscopy. After $9 \mathrm{~d}$ cells were washed twice with $1 \times$ PBS, and total RNA was extracted using the RNeasy kit (QIAGEN Inc., Chatsworth, CA). $3 \mu \mathrm{g}$ RNA was reverse transcribed in the presence of $1 \mu \mathrm{g}$ oligo(dT) and $50 \mathrm{ng}$ random hexanucleotide primers using Superscript II RNase H- Reverse Transcriptase (Life Technologies, Inc.). PCR was carried out as described above after validation that under these conditions PCR product accumulation remained in the exponential phase using $200 \mathrm{nM}$ of each sense and antisense primer. Isoform-specific sense oligonucleotides for nNOSa (A1 5'-agcgggatccacagccetggaact- $3^{\prime}$ ), nNOSb (B1 5'-gactgaggggcgacactaccatgc- $\left.3^{\prime}\right)$, and nNOSc (C1 5'-caccacagctctggaatgaaaga- $3^{\prime}$ ), respectively, were combined with the common antisense primer N1. A cDNA pool derived from brain, embryo, and kidney was included as positive control. To monitor expression of a housekeeping gene exon spanning oligonucleotides of the rat $\beta$-actin gene (ract1 5'-gaccttcaacacccagccatg-3'; ract2 5' -gggccggactcatcgtactcct- $\left.3^{\prime}\right)$ and were used to amplify a 726-bp fragment during 25 cycles of PCR (21). Amplicons were fractionated on $2 \%$ agarose gels and blotted onto nylon membrane. Southern blot analysis was performed with the internally located oligonucleotide N3 labeled with $\left[\gamma^{-32} \mathrm{P}\right]$ ATP using T4 polynucleotide kinase (New England Biolabs Inc., Beverly, MA).

Screening of a rat yeast artifical chromosome library. PCR amplification of screening pools of a rat YAC library (22) was carried out using oligonucleotide sets specific for the alternative first exons of nNOSa (Ay1 5'-ggcgctgttggagcccggagca-3'; Ay2 5'-ctgctgccgagcccgcgcagtt- $3^{\prime}$ ), and nNOSb (By1 5'-ccttgagtcgctcggccgcaccat-3'; By2 $5^{\prime}$-ggactcctgcgaagccgtccettg- $\left.3^{\prime}\right)$ as well as for the $5^{\prime}$ end (5end $15^{\prime}$ cgtctgacaagctggtgaccaaga- $3^{\prime}$; 5end $25^{\prime}$-gaacctccagggcactgtcatagc- $3^{\prime}$ ) and $3^{\prime}$ end (3end1 $5^{\prime}$-ggctctcacaaaaccgcatcctc-3'; 3end2 5' -aaagagggtggttgggggcttaa- $\left.3^{\prime}\right)$ of the published nNOS cDNA sequence. A single YAC, $228 \mathrm{H} 7$, was found that yielded specific amplification products for all primer sets tested. YAC DNA was isolated from yeast cells embedded in agarose plugs (22) and digested overnight with each of the restriction endonucleases Pac I, Pme I, Sfi I, and Sap I (New England Biolabs) or with combinations thereof. Subsequently, plug DNA was size-fractionated by pulsed field gel electrophoresis in a $1 \%$ agarose gel in $0.5 \times \mathrm{TBE}$ at $14^{\circ} \mathrm{C}, 6 \mathrm{~V} / \mathrm{cm}$ for $18 \mathrm{~h}$ with a switching time of $15 \mathrm{~s}$ and blotted onto nylon membrane. Total rat genomic DNA was included as control. Southern blot analysis was performed with probes generated by PCR using the same primers as for the library screening. Probes were labeled with $\left[\alpha-{ }^{32} \mathrm{P}\right] \mathrm{dCTP}$ (DuPont $\mathrm{NEN}$ ) in the presence of random hexamers using T7 DNA polymerase (Pharmacia Diagnostics AB).

\section{Results}

Isolation of multiple $n N O S$ transcripts with unique 5'UTRs. Cloning and sequencing of cDNA by RACE from brain, kidney, heart, intestine, and embryo led to the identification of three different nNOS mRNA species that are designated as nNOSa, nNOSb, and nNOSc. nNOSa was overall the most abundant isoform, found especially in the brain, while $\mathrm{nNOSb}$ was almost exclusively expressed in embryonal tissue and nNOSc in the kidney. A common $3^{\prime}$ terminal sequence consisting of $30 \mathrm{bp}$ was found to be shared by all isoforms extending $5^{\prime}$ from position +2 of the published sequence of the rat nNOS cDNA (3). Based on PCR analysis of genomic DNA, this 30-bp common sequence is contiguous with the published $5^{\prime}$ end of the gene, and therefore represents the actual $5^{\prime}$ end of the second exon (previously perceived as the first exon) of the rat nNOS gene. The unique additional $5^{\prime}$ terminal sequences represent differential exon 1 sequences of $\mathrm{nNOSa}, \mathrm{nNOSb}$ and nNOSc that are spliced to this newly identified 30-bp domain of the second exon (Fig. 1). The newly identified unique first exons of nNOSa, nNOSb, and nNOSc are 442, 134, and $151 \mathrm{bp}$ in length, respectively. RT-PCR with isoform-specific sense and antisense primers located at various positions of the cDNA as well as direct sequencing of PCR products yielded no evidence for additional nNOS mRNA species or further variations within the coding sequence, except for a previously described 102-bp insert between exon 16 and 17 (23) that was detectable in skeletal muscle and embryo (data not shown). Comparative databank searches using the BLAST module showed that nNOSa shares a high degree of homology with one of the two alternative first exons identified in the mouse, while neither nNOSb nor nNOSc showed homology to any of the other alternative first exons described in mouse or human $(17,18)$.

Tissue- and development-specific expression of $n N O S$ mRNAisoforms. The expression pattern of the different transcriptional variants was determined by RNase protection assay using cDNA clones obtained by RACE as probes that included part of the published sequence corresponding to position +2 


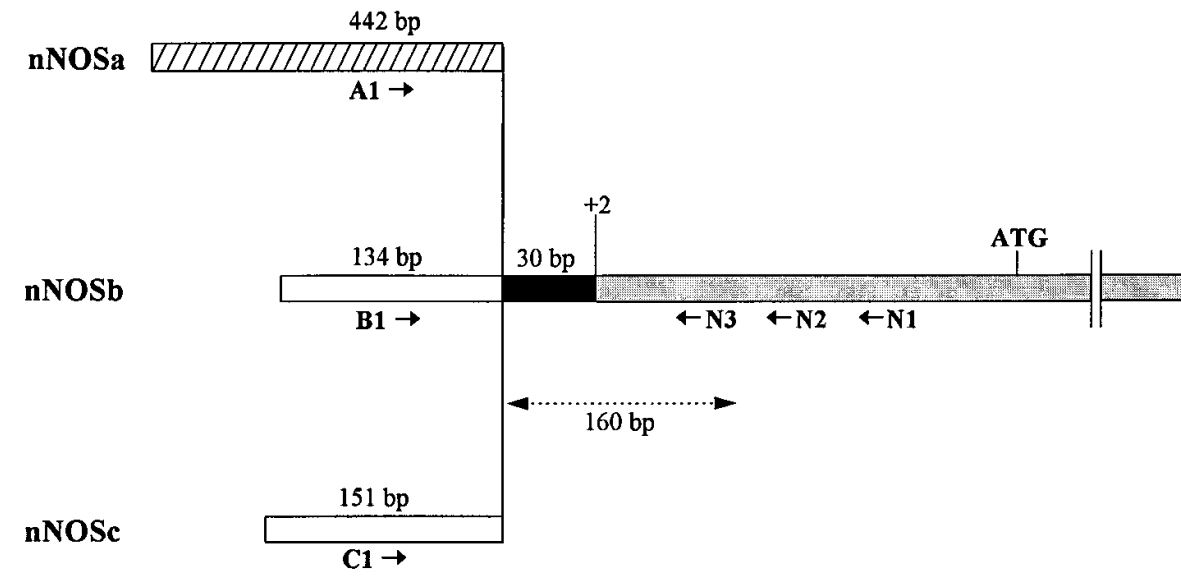

B

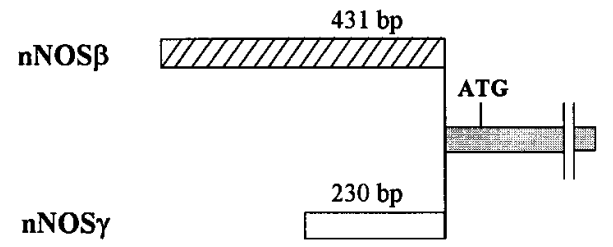

C

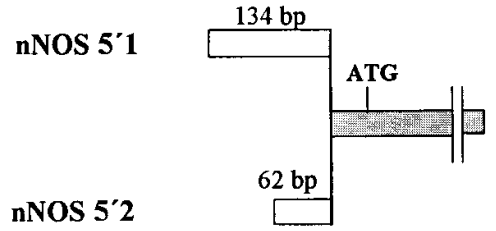

Figure 1. Schematic presentation of alternative first exons in rat, mouse, and human nNOS gene. (A) The light grey bar denotes the published nNOS cDNA sequence of the rat containing the translation initiation site (ATG). A 30-bp extension from position $2(+2)$ of the published sequence common to all alternative 5'UTRs is indicated by a dark grey bar. Unique sequences of the alternative 5'UTRs are indicated as hatched bar (nNOSa) or open bars (nNOSb, nNOSc). A1, $B 1$, and $C 1$ : sense primer used for RT-PCR; N1, N2, and N3: antisense primer used for RACE and RT-PCR. Clones used in RNase protection assays included a 160-bp stretch common to all nNOS isoforms. Alternative 5' sequences of the mouse nNOS gene (nNOS $\beta$ and $\mathrm{nNOS} \gamma ; B)$ and of the human nNOS gene (nNOS5'1 and nNOS5'2; $C$ ). Homologous first exons (nNOSa of the rat and $\mathrm{nNOS} \beta$ of the mouse) are indicated by hatched bars. to +131 . Because all three nNOS mRNA isoforms shared the additional common, newly recognized stretch of $30 \mathrm{bp}$ in exon 2, a 160-bp fragment was expected to be protected, and was indeed found to be present in all tissues expressing any of the nNOS variants. In addition, specific transcript signals corresponding to the $5^{\prime}$ terminus of each nNOSa, nNOSb, and nNOSc, were found depending on tissue-specific isoform expression. Consistent with its high prevalence among RACE clones, nNOSa was the most abundant isoform. It showed highest expression in brain, followed by kidney, intestine, E18 embryo, adrenal, heart, and skeletal muscle. In contrast, nNOSb was exclusively expressed in E18 embryo, while nNOSc expression was found in kidney, skeletal muscle, and embryo (Fig. 2). Absolute expression levels of nNOSc are low compared to the other isoforms, and signals were detectable only after prolonged exposure to autoradiographic material. The only tissue that consistently tested negative for all three isoforms of nNOS was the testis.

Induction of $n N O S a$ in terminally differentiated PC12 cells. Using sense primers A1, B1, and C1 specific for the alternative first exons in combination with the antisense primer
N1 specific for exon 2 of the rat nNOS gene, RT-PCR followed by Southern blotting was carried out on cDNA obtained from rat pheochromocytoma-derived PC12 cells before and after induction of neuronal differentiation by NGF. While nNOS expression was almost undetectable under basal conditions, strong induction of nNOSa mRNA was seen in differentiating cells (Fig. 3).

Physical mapping of the alternative first exons of the rat $n N O S$ gene. PCR analyis using genomic DNA as template showed that all newly identified 5'UTRs are encoded by a single exon, and are not interrupted by intronic sequences. Using primers anchored in exons $1 \mathrm{c}$ and $1 \mathrm{~b}$, the two exons were localized at a distance of $150 \mathrm{bp}$ from each other, with exon 1c positioned upsteam of exon $1 \mathrm{~b}$. PCR pools of the rat YAC library were screened with primer pairs specific for the alternatively spliced exons (primer pairs Ay1/Ay2 for exon 1a and By1/By2 for exons $1 \mathrm{~b}$ and $1 \mathrm{c}$ ), as well as for the $5^{\prime}$ end (exon 2) and $3^{\prime}$ end of the published cDNA sequence. A single YAC (1.1 Mb) was found that gave rise to amplification products from all primer sets. Restriction mapping using infrequently cutting restriction endonucleases positioned exon $1 \mathrm{a}$ at a distance of at 


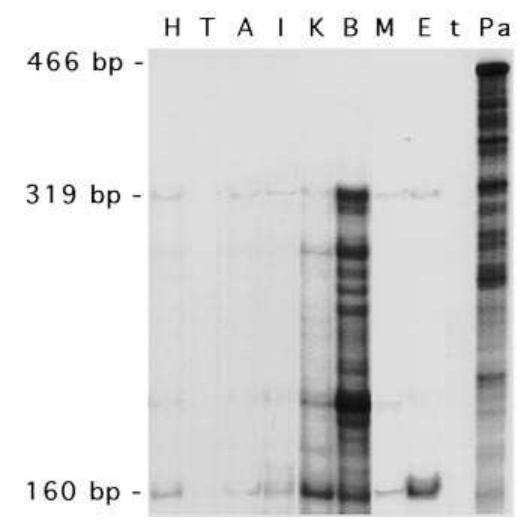

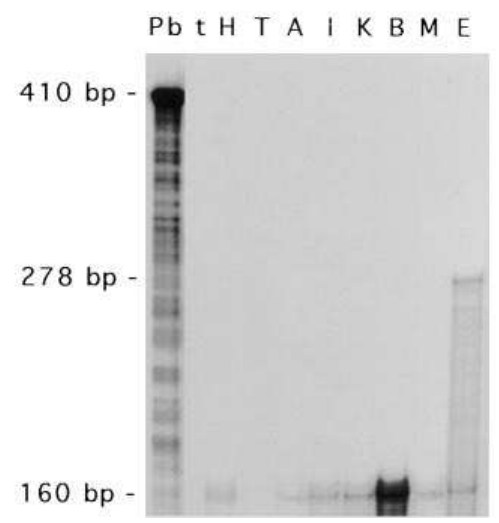

C

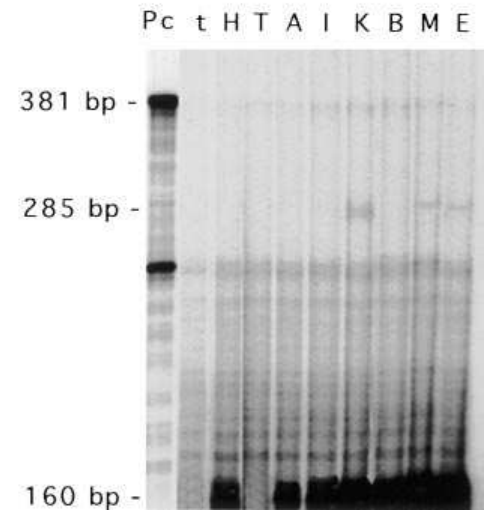

Figure 2. Analysis of expression by RNase protection assay. Expression patterns of nNOSa $(A), \mathrm{nNOSb}(B)$, and nNOSc $(C)$ were examined using $100 \mu \mathrm{g}$ total RNA from heart $(H)$, testis $(T)$, adrenal $(A)$, intestine $(I)$, kidney $(K)$, brain $(B)$, skeletal muscle $(M)$, and E18 embryo $(E)$; $t$, tRNA. The sizes of the protected fragments for nNOSa, nNOSb, and nNOSc were $319 \mathrm{bp}, 278 \mathrm{bp}$, and $285 \mathrm{bp}$, respectively. $P a, P b$, and $P c$ indicate in vitro-transcribed probes that are 466, 410, and $381 \mathrm{bp}$, respectively, and included vector-encoded sequence. A protected fragment $160 \mathrm{bp}$ in size corresponds to the common sequence shared by all isoforms.

least $15 \mathrm{~kb}$, but of not more than $20 \mathrm{~kb}$ upstream of exon 2. Exons $1 \mathrm{~b}$ and $1 \mathrm{c}$ were placed approximately an additional $40 \mathrm{~kb}$ upstream of exon 1a (Fig. 4).

\section{Discussion}

Our results demonstrate that nNOS in the rat occurs in at least three isoforms characterized by the presence of different first

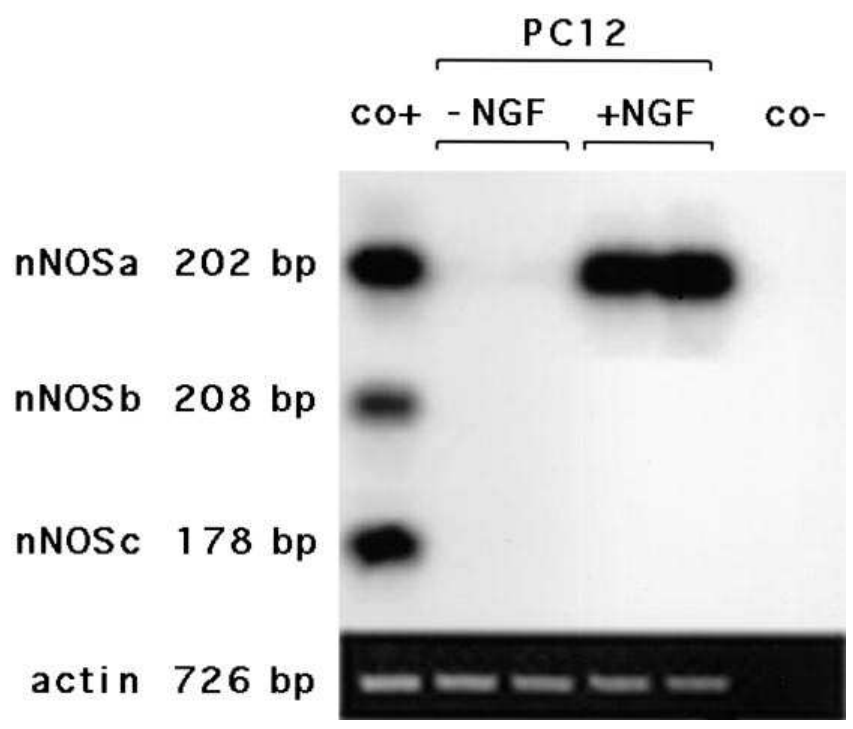

Figure 3. Induction of nNOSa in terminally differentiated PC12 cells. RT-PCR of RNA isolated from each of two dishes of PC12 cells without $(-N G F)$ and with NGF treatment $(+N G F)$. Sense primers A1, $\mathrm{B} 1$, and $\mathrm{C} 1$ (specific for $\mathrm{nNOSa}, \mathrm{nNOSb}$, and nNOSc, respectively) were combined with antisense primer N3 (see Fig. 1), and yielded fragment sizes of 202, 208, and $187 \mathrm{bp}$, respectively. $\mathrm{Co}+$, positive control (cDNA pool derived from rat brain, kidney and embryo). $\mathrm{Co}-$, negative control $\left(\mathrm{H}_{2} \mathrm{O}\right)$. Actin, 726-bp fragment of the rat $\beta$-actin cDNA. exons, and that the previously published information regarding the $5^{\prime}$ end of what we now recognize as exon 2 of the rat nNOS cDNA was incomplete. In addition, we report that the three isoforms of the gene show markedly different expression patterns that are spatially and developmentally distinct. While the relative abundance of nNOS mRNA in the tissues analyzed is consistent with previous studies, our findings demonstrate that overall mRNA levels in some tissues are composed of different amounts of the mRNA variants of nNOS. Further studies using in situ hybridization are necessary to elucidate the spatial and temporal expression of nNOS-mRNA on a tissular and cellular level. These observations suggest a more complex and intricate pattern of modulation of expression for this enzyme than previously appreciated, and challenge the heretofore held perception that nNOS is a constitutively expressed gene.

The newly identified first exons $1 \mathrm{a}, 1 \mathrm{~b}$, and $1 \mathrm{c}$ of the rat nNOS gene are located upstream of the coding sequence, and therefore, do not result in a modification of the encoded protein. Recently, two nNOS mRNAs with alternative 5'UTRs were identified in the mouse as well as in humans $(17,18)$. While rat nNOSa shows close homology to one of the mouse mRNA isoforms (nNOS $\beta$ ), however, no similarity was detectable between nNOSb and nNOSc and the second 5'UTR $(\mathrm{nNOS} \gamma)$ in the murine nNOS gene. Likewise, no homology was found between any of the three first exons in the rat and the two reported alternative exons in humans (nNOS $5^{\prime}$ and nNOS 5'2). This observation may simply reflect species-specific differences, or point to the presence of further yet to be identified alternative $5^{\prime}$ ends in the rat that are homologous with the alternative first exon in mouse and the two human isoforms, and that may have escaped our present 5'RACE analysis due to low abundance. Although the heterogeneity of the $5^{\prime}$ ends of nNOS mRNA could be explained by alternative splicing events, it is likely that activation of differential promoters is involved in their generation. In support of this, the two human nNOS mRNA species are thought to be transcribed from two closely linked, but separable promoters (17). 
$\mathbf{A}$

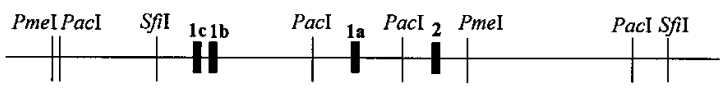

$\overline{10 \mathrm{~Kb}}$

$\mathbf{B}$

exon 1c

ATTCCGATCC AGCAGGCATG GCGTAGACAC AGAAGATTCA GGGCTGCGAT CCTGACTCCA GGCCATCCCA GCCAGCTGGG GGAAACCTGA GCCACCTCTA TTCCCTGGCA GAAGCTGCCA CAACCACCAC AGCCTCTGGA ATGAAAGAAA Ggtcagagte tagagaagca gggctggcgc gatggaggga gggctgccgg ggtgetgget agccaccetc acctctctec tctctgctt cetggggaag cetgagtacc ctgagcetgg gtgtgeccec cetgeccagg gettggectc tgggeccegt getgagacge etcceagcet gecectgggg aggggecacC TGGTGTCAAT TAAGGCCTTG AGTCGCTCGG CCGCACCATG CAGGGCTGCT GATTAGAGCT GCAGTCTCTC CAGATGGGGA GCACGGACTG AGGGGCGACA CTACCATGCC AAGGGACGGC TTCGCAGGAG TCC

GTGACCACTA ATTCGTTTCA GCGGTGATAG GATAAAGCAG GGACATTAAG AAATAAATTC CCCTTCACGA CCITCGCGGA GCTCGCTGCG CGGTTCCTGC ATATTGATGC AGCCGCAGGG TGCGAGCGGC TACTTAGCAC AGAGACTGCT CCTAGGGGCA GCTGCGGCCG GGGTGAGCCA GGTTGTGCGA AGGGAAGCTG GCAGCCTCCA GTCCTGAACT TEGCTGGGCT AGAGCGGAGC GAGGCGGGAG CAAGAGACTG GGAGGGGGCG GGGAGCGCTC GCTCTGGAAG CTCCTACCCT GTGGCGCTGT TGGAGCCCGG AGCATCCTGC CCCGGAGCGA GGCTCTGGAT CCGCGCGCTC AGCAGCTGCG GGAGGAGGAG GCGCGGAAGA CAGGGTGATC AGCGGGATCC ACAGCCCTGG AACTGCGCGG GCTCGGCAGC AG

exon 2 CTCCAGGTAG AAGCCCCTAT GTCACCTCTG

C

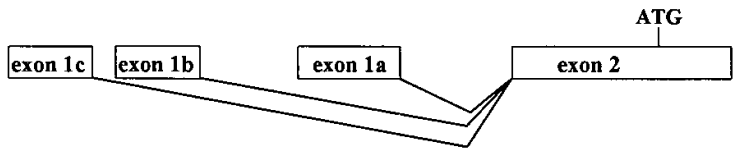

Figure 4. Physical mapping of the rat nNOS gene by pulsed-field gel electrophoresis. $(A)$ Restriction map of YAC 228H7 with infrequently cutting restriction endonucleases and relative position of probes for exon 1a, 1b, and 1c. (B) Sequences for the three alternative exons as well as for the newly identified $5^{\prime}$ end of exon 2 are depicted in capital letters. Lower case letters denote the intervening sequence between exon $1 \mathrm{c}$ and $1 \mathrm{~b}$. $(C)$ Schematic presentation of the splicing pattern of the alternative $5^{\prime}$ UTRs of the rat nNOS gene. Drawings are not to scale.

Furthermore, the alternative use of multiple first exons flanked by tissue-specific promoters has been demonstrated for a number of other genes that are ubiquitously expressed, such as for the gene encoding aromatase (which, like nNOS, is a member of the cyotchrome P-450 superfamily) (24) and the gene encoding the glucocorticoid receptor (24). The hypothesis that expression of nNOSb may be driven by a developmentally regulated promoter is consistent with its transient and exclusive expression during development $(14,15)$. Our observation of developmentally specific expression patterns may shed some light on recent observations obtained in mice carrying a deletion of exon 2 of the nNOS gene (25). We speculate that the relative absence of developmental pathology in this model may be explained by predominance of the still functional transcript nNOS $\beta$ from the first alternative exon, that retains $85 \%$ activity during critical phases of development $(18,25)$. The presence of multiple promoters would allow extensive regulation of transcription of a single gene both spatially and temporally, as suggested by the tissue- and development-specific expression pattern of nNOS mRNA species presented here.

Reminiscent of the two alternative first exons described in the human nNOS gene, exons $1 \mathrm{c}$ and $1 \mathrm{~b}$ are located in close proximity to each other. A pattern search for the sequence juxtaposed between exons $1 \mathrm{c}$ and $1 \mathrm{~b}$ revealed the presence of a number of consensus sequences such as those known to interact with nuclear factor-1, AP1, MyoD, GATA, and histoneH4. Any critical assessment concerning the functional relevance of these sequence elements and the characterization of putative promoters, however, must await the results of specific experiments.

Given the broad distribution of nNOS throughout the body and the extensive spectrum of biological functions mediated by NO, it would seem likely that the expression of this enzyme is regulated in a differentiated fashion. The use of alternative promoters is one mechanism that allows spatial- and temporalspecific regulation of transcription. The resulting complexity on the transcriptional level affords the organism the capacity to respond to numerous signals with a high degree of specificity. Thus, the level of the short-lived molecule NO in any given tissue is dependent not only on the calcium concentration, but also on a cell's specific endowment of trans-acting factors that control the relative level of expression of each of the known NO-producing enzymes. The identification of cis- and transacting factors that are responsible for the expression of the transcriptional variants of the nNOS gene will provide insight into the molecular mechanisms that control nNOS expression, and thus into the diversity of functional properties of nNOS in these tissues.

Increasing evidence suggests a role of $5^{\prime}$ and $3^{\prime} \mathrm{UTR}$ of mRNAs in the regulation of gene expression. Thus, so-called iron-responsive elements, found in 5'UTRs of genes involved in iron metabolism control their translational rate in response to changes of iron levels (26). Certain sequences within the $3^{\prime}$ UTR have been shown to affect mRNA transport and stability (27), and short open reading frames within 5'UTR have been implicated in the inhibition of translation (28). It is therefore certainly possible that variations in length or sequence of the 5'UTRs of the rat nNOS mRNA isoforms may affect nNOS protein levels via different posttranscriptional mechanisms.

NO has an extremely wide spectrum of functional properties that is unprecedented in biology. As one of the smallest water- and lipid- soluble carriers capable of chaperoning a free electron within and between cells, NO has access to a number of molecules, and is able to modify the state of active sites. It has been implicated in the regulation of ion channels, GTPases, and transcription factors (29-31). To fulfill these multiple roles, a regulated mode of expression of nNOS, as supported by our findings, would appear reasonable, and, indeed perhaps essential.

Our observation of developmentally specific expression patterns of nNOS isoforms is compatible with emerging evidence that $\mathrm{NO}$ is involved in developmental processes such as apoptosis and differentiation. NO has been shown to trigger the switch to growth arrest during differentiation of PC12 cells into neuronal cells in response to NGF, a phenomenon that is accompanied by an induction of both calcium-independent and calcium-dependent NOS acitivity (20). Likewise, NO has been implicated as conferring antiproliferative effects during 
Drosophila development, controlling the balance between cell proliferation and cell differentiation (32). Although the relevant NOS isozyme has not been specifically identified, dNOS, the only NOS-encoding gene cloned from Drosophila to date, shows strong homology to the mammalian nNOS (33). Our finding that differentiation of $\mathrm{PC} 12$ cells into neurons is accompanied by the induction of nNOSa, the most abundant nNOS transcript found in the mature brain, is consistent with this proposed growth- or differentiation-regulating role of nNOS. In addition, since transcriptional activation of specific promoter elements by NGF in PC12 cells has been demonstrated for a number of genes, such as the genes encoding neuropeptide $\mathrm{Y}$ gene and transforming growth factor-beta gene $(34,35)$, it seems reasonable to speculate that a similar mechanism may account for the specific induction of nNOSa.

In summary, we report original evidence that differential transcripts of nNOS show tissue- and development-specific expression patterns, and propose that this may represent a heretofore unrecognized mechanism of regulatory modulation of nNOS expression.

\section{Acknowledgments}

We are grateful to Bradford D. Verrecchia and Lily Eng for excellent technical help. This work was supported by a Research Career Development Award (K04-HL03138-01) to K. Lindpaintner, and a fellowship Le1074/1-1 by the German Research Council (Deutsche Forschungsgemeinschaft) to M.A. Lee.

\section{References}

1. Lamas, S., P.A. Marsden, G.K. Li, P. Tempst, and T. Michel. 1992. Endothelial nitric oxide synthase: molecular cloning and characterization of a distinct contitutive enzyme isoform. Proc. Natl. Acad. Sci. USA. 89:6348-6352.

2. Marsden, P.A., H.H.Q. Heng, S.W. Scherer, R.J. Stewart, A.V. Hall, X. Shi, L. Tsui, and K.T. Schappert. 1993. Structure and chromosomal localization of the human constitutive endothelial nitric oxide synthase gene. J. Biol. Chem. 268:17478-17488.

3. Bredt, D.S., P.M. Hwang, C.E. Glatt, C. Lowenstein, R.R. Reed, and S.H. Synder. 1991. Cloned and expressed nitric oxide synthase structurally resembles cytochrome P-450 reductase. Nature (Lond.). 351:714-718.

4. Hall, A.V., H. Antoniou, Y. Wang, A.H. Cheung, A.M. Arbus, S.L. Olson, W.C. Lu, C. Kau, and P.A. Marsden. 1994. Structural organization of the human neuronal nitric oxide synthase gene (NOS1). J. Biol. Chem. 269:3308233091.

5. Xie, Q.-W., H.J. Cho, J. Calaycay, R.A. Mumford, K.M. Swiderek, T.D. Lee, A. Ding, T. Troso, and C. Nathan. 1992. Cloning and characterization of inducible nitric oxide synthase from mouse macrophages. Science (Wash. DC). 256:225-228.

6. Chartrain, N.A., D.A. Geller, P.P. Koty, N.F. Sitrin, A.K. Nussler, E.P. Hoffman, T.R. Billiar, N.I. Hutchinson, and F.B. Mueller. 1994. Molecular cloning, structure, and chromosomal localization of the human inducible nitric oxide synthase gene. J. Biol. Chem. 269:6765-6772.

7. Nathan, C. and Q.-W. Xie. 1994. Nitric oxides synthases: roles, tolls, and controls. Cell. 78:915-918.

8. Lowenstein, C.J., E.W. Alley, P. Raval, A.M. Snowman, S.H. Snyder, S.W. Russel, and W.J. Murphy. 1993. Macrophage nitric oxide synthase gene: two upstream regions mediate induction by interferon gamma and lipopolysaccharide. Proc. Natl. Acad. Sci. USA. 90:9730-9734.

9. Sessa, W.C., J.K. Harrison, D.R. Luthin, J.S. Pollock, and K.R. Lynch. 1993. Genomic analysis and expression patterns reveal distinct genes for endothelial and brain nitric oxide synthase. Hypertension (Dallas). 21:934-938.

10. Wilcox, C.S., W.J. Welch, F. Murad, S.S. Gross, G. Taylor, R. Levi, and H.H.H.W. Schmidt. 1992. Nitric oxide synthase in macula densa regulates glomerular capillary pressure. Proc. Natl. Acad. Sci. USA. 89:11993-11997.

11. Weiner, C.P., I. Lizosoain, S.A. Baylis, R.G. Knowles, I.G. Charles, and S. Moncada. 1994. Induction of calcium-dependent nitric oxide synthases by sex hormones. Proc. Natl. Acad. Sci. USA. 91:5212-5216.

12. Verge, V.M., Z. Xu, X.J. Xu, Z. Wiesenfeld-Hallin, and T. Hokfelt 1992. Marked increase in nitric oxide synthase mRNA in rat dorsal root ganglia after peripheral axotomy: in situ hybridisation and functional studies. Proc. Natl. Acad. Sci. USA. 89:11617-11621.

13. Herdegen, T., S. Brecht, B. Mayer, J. Leah, W. Kummer, R. Bravo, and M. Zimmermann. 1993. Long-lasting expression of jun and krox transcription factors and nitric oxide synthase in intrinsic neurons of the rat brain following axotomy. J. Neurosci. 13:4130-4145.

14. Bredt, D.S. and S.H. Snyder. 1994. Transient nitric oxide synthase neurons in embryonic cerebral cortical plate, sensory ganglia, and olfactory epithelium. Neuron. 13:301-313.

15. North, A.J., R.A. Star, T.S. Brannon, K. Ujiie, C. Welsch, C.J. Lowenstein, S.H. Snyder, and P.W. Shaul. 1994. Nitric oxide synthase type I and type III gene expression are developmentally regulated in rat lung. Am. J. Physiol. 266:L635-L641.

16. Williams, C.V., D. Nordquist, and S.C. McLoon. 1994. Correlation of nitric oxide synthase expression with changing patterns of axon projections in the developing visual system. J. Neurosci. 14:1746-1755.

17. Xie, J., P. Roddy, T.K. Rife, F. Murad, and A.P. Young. 1995. Two closely linked but separable promoters for human neuronal nitric oxide synthase gene transcription. Proc. Natl. Acad. Sci. USA. 92:1242-1246.

18. Brennan, J.E., D.S. Chao, S.H. Gee, A.W. McGee, S.E. Craven, D.R Santillano, Z. Wu, F. Huang, H. Xia, M.F. Peters, et al. 1996. Interaction of nitric oxide synthase with the postsynaptic density protein PSD-95 and a1-syntrophin mediated by PDZ Domains. Cell. 84:757-767.

19. Frohman, M.A., M.K. Dush, and G.R. Martin. 1988. Rapid production of full-length cDNAs from rare transcripts: amplification using a single genespecific oligonucleotide primer. Proc. Natl. Acad. Sci. USA. 85:8898-9002.

20. Peunova, N. and G. Enikolopov. 1995. Nitric oxide triggers a switch to growth arrest during differentiation of neuronal cells. Nature (Lond.). 375:68-73.

21. Nudel, U., R. Zakut, M. Shani, S. Neuman, Z. Levy, and D. Yaffe. 1983. The nucleotide sequence of the rat cytoplasmic beta-actin gene. Nucleic Acids Res. 11:1759-1771.

22. Cai, L., L.C. Schalkwyk, A. Schoeberlein-Stehli, R.Y.L. Zee, A. Smith, T. Haaf, M. Georges, H. Lehrach, and K. Lindpaintner. 1997. Construction and characterization of a 10-genome equivalent yeast artificial chromosome library for the laboratory rat, rattus norvegicus. Genomics. 39:385-392.

23. Silvagno, F., H. Xia, and D.S. Bredt. 1996. Neuronal nitric oxide synthase $\mu$, an alternatively spliced isoform expressed in differentiated skeletal muscle. J. Biol. Chem. 271:11204-11208.

24. Harada, N., T. Utsumi, and Y. Takagi. 1993. Tissue-specific expression of the human aromatase cytochrome P-450 gene by alternative use of multiple exons 1 and promoters, and switching of tissue-specific exons 1 in carcinogenesis. Proc. Natl. Acad. Sci. USA. 90:11312-11316.

25. Huang, P.L., T.M. Dawson, D.S. Bredt, S.H. Snyder, and M.C. Fishman. 1993. Targeted disruption of the neuronal nitric oxide synthase gene. Cell. 75 : 1273-1286.

26. Hentze, M.W., and L.C. Kühn. 1996. Molecular control of vertebrate iron metabolism: mRNA-based regulatory circuits operated by iron, nitric oxide, and oxidative stress. Proc. Natl. Acad. Sci. USA. 93:8175-8182.

27. Atwater, J.A., R. Wisdom, and I.M. Verma. 1990. Regulated mRNA stability. Annu. Rev. Genet. 24:519-541.

28. Zimmer, A., A.M. Zimmer, and K. Reynolds. 1994. Tissue specific expression of the retinoic acid receptor-beta 2 : regulation by short open reading frames in the 5-coding region. J. Cell Biol. 127:1111-1119.

29. Bolotina, V.M., S. Najibi, J.J. Palacino, P.J. Pagano, and R.A. Cohen. 1994. Nitric oxide directly activates calcium-dependent potassium channels in vascular smooth muscle. Nature (Lond.). 368:850-853.

30. Lander, H.M., P.K. Sehajpal, and A. Novogrodsky. 1993. Nitric oxide signaling; a possible role for G-proteins. J. Immunol. 151:7182-7187.

31. Peunova, N., and G. Enikolopov. 1993. Amplification of calcium-induced gene transcription by nitric oxide in neuronal cells. Nature (Lond.). 364:450-453.

32. Kuzin, B., I. Roberts, N. Peunova, and G. Enikolopov. 1996. Nitric oxide regulates cell proliferation during Drosophila development. Cell. 87:639649

33. Regulski, M., and T. Tully. 1995. Molecular and biochemical characterization of dNOS: a Drosophila $\mathrm{Ca}^{2+}$ /calmodulin-dependent nitric oxide synthase. Proc. Natl. Acad. Sci. USA. 92:9072-9076.

34. D'Arcangelo, G., R. Habas, S. Wang, S. Halegoua, and S.R. Salton. 1996. Activation of codependent transcription factors is required for transcription induction of the vgf-gene by nerve growth factor and ras. Mol.Cell.Biol. 16: 4621-4631.

35. Kim, S.J., K. Park, B.B. Rudkin, B.R. Dey, M.B. Sporn, and A.B. Roberts. 1994. Nerve growth factor induces transcription of transforming growth factor-beta through a specific promoter element in PC12 cells. J. Biol. Chem. 269:3739-3744. 